\title{
Mesoporous Assembly of Layered Titanate with Well-Dispersed Pt Cocatalyst
}

\author{
Tae Sung Jung, ${ }^{\text {,b }}$ Tae Woo Kim, ${ }^{a}$ and Seong-Ju Hwang \\ Center for Intelligent Nano-Bio Materials (CINBM), Department of Chemistry and Nano Sciences, \\ Ewha Womans Lnversity, Seoul 120-750, Korea. E-mail: hw'angsjuatewha.ackr \\ Received December 8, 2008. Accepted January 30, 2009
}

\begin{abstract}
A mesoporous assembly of layered titanate with well-dispersed Pt cocatalysts has been synthesized via a restacking of exfoliated titanate nanosheets and a simultaneous adsorption of Pt nanoparticles. According to powder X-ray diffraction analy'sis, the obtained mesoporous assembly shows amorphous structure corresponding to the disordered stacking of layered titanate crystallites. Field enission-scanning electron microscopy and $\mathrm{N}_{2}$ adsorption-desorption isotherm measurement clearly demonstrate the formation of mesoporous structure with expanded surface area due to the house-of-cards type stacking of the titanate crystallites. From high resolution-transmission electron microscopy and elemental mapping analyses, it is found that Pt nanoparticles with the size of $-2.5 \mathrm{~nm}$ are homogeneously dispersed in the mesoporous assembly of layered titanate. In comparison with the protonated titanate, the present mesoporous assembly of layered titanate exhibits better photocatalytic activity for the photodegradation of organic molecules. This finding underscores that the restacking of exfoliated nanosheets is quite useful not only in creating mesoporous structure but also in improving the photocatalytic activity of titanium oxide.
\end{abstract}

Key Words: Mesoporous assembly. Restacking. Surface expansion. Layered titanate. Photocatalysts

\section{Introduction}

Over the past decades. titanium oxide has been one of the most investigated photocatalysts because of its many advantages such as low price. low toxicity, high stability, and so on. ${ }^{1,2}$ The application of titanium oxide ranges from pollution clean-ups to antiseptic or hygienic purposes. because this material can induce effective photodegradation of various organic or bio-molecules including industrial pollutants. bacteria, and viruses. ${ }^{1.2}$ To dates, many attempts have been made to improve the photocataly tic activity of titanium oxide ${ }^{2-6}$ Taking into account the fact that photocatalytic reaction occurs on the surface of photocatalyst, the increase of surface area would be effective in improving the photocatalytic activity of titanium oxide. Recently we have found that the exfoliated nanosheets of layered titanate are very useful precursors for synthesizing hierarchically assembled superstructures with diverse functionalities. ${ }^{72}$ The disordered restacking of exfoliated titanate nanosheets is considered to produce mesoporous assembly of layered titanium oxide with expanded surface area. Such an expansion of surface area upon the restacking process would enhance the photocataly tic activity of titanium oxide. Furthermore. the formation of porous structure is advantageous in adsorbing Pt cocatalysts homogeneously onto the photocatalyst without significant agglomeration.

In this study, we have successfully prepared the mesoporous assembly of layered titanate nanosheets with well-dispersed Pt cocatalysts. The crystal structure, crystal morphology, and chemical composition of the obtained mesoporous titanate

\footnotetext{
${ }^{3}$ These two authors contributed equally to this work.

'Present address: Korean Minjok Leadership Academy, Gangwon 225-823, Korea
}

have been systematically characterized using powder X-ray diffraction (XRD). high resolution-transmission electron microscopy (HR-TEM). field enission-scanning electron microscopy (FE-SEM), and elemental mapping analysis. respectively. The formation of mesoporous structure was verified by $\mathrm{N} z$ adsorption-desorption isotherm measurement. Also. we have studied the photocatalytic activity of the present mesoporous titanate by monitoring the photodegradation of methylene blue (MB) under UV-vis irradiation.

\section{Experimental Section}

Preparation of Exfoliated Titanate Nanosheets. The host cesium titanate. $\mathrm{Cs}_{1,6}: \mathrm{Ti}_{1.83} \square_{1,1.1}: \mathrm{O}_{4}$. was prepared by heat-treatment for the stoichiometric nuixture of $\mathrm{TiO}_{3}$ and $\mathrm{Cs}_{2} \mathrm{CO}_{3}$ at $800{ }^{\circ} \mathrm{C}$ for $40 \mathrm{~h}$. as reported previously. It protonated derivative was obtained by the reaction between the pristine

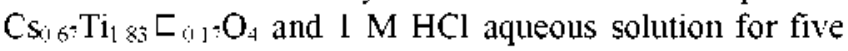
day's. The $\mathrm{HCl}$ solution was refreshed for every day. The colloidal suspension of exfoliated titanate nanosheets was prepared by the reaction between the protonated titanate and excess TBA solution (tetrabutylammonium, $40 \%$ ) for 14 days. After the reaction. a small fraction of incompletely exfoliated particles were separated by centrifugation at $12.000 \mathrm{rpm}$.

Preparation of Positive-Charged Pt Nanoparticles. Monodisperse platinum nanoparticles were prepared by reacting 5 $\mathrm{mL}$ of $5.0 \mathrm{mM} \mathrm{H} \mathrm{H}_{2} \mathrm{PtCl}_{4} \cdot 6 \mathrm{H}_{2} \mathrm{O}$ aqueous solution. $2.8 \mathrm{mg}$ of PVP (polyvinyl pyrrolidone. $\mathrm{MW}=40.000$ ). and $48 \mathrm{~mL}$ of nixed methanol/ $\mathrm{H}_{-} \mathrm{O}$ solvent $(9 / 1, \mathrm{v} / \mathrm{v})$ at $80^{\circ} \mathrm{C}$ under refluxing with vigorous stirring. according to the previously reported method. ${ }^{15}$ The polymer PVP was used as protecting agent to limit the crystal growth of Pt particles to nanometer-scale. ${ }^{13}$ After the reaction for $2 \mathrm{~h}$, the yellow chloroplatinic acid was changed into dark brown-colored powder, as shown in Figure 
1. The complete reduction of the $\left[\mathrm{PtCl}_{6}\right]^{3 \cdot}$ ion into Pt metal was further confinned by UV-vis results showing that an absorption peak at $-260 \mathrm{~nm}$ corresponding to $\left[\mathrm{PtCl}_{6}\right]^{{ }^{-}}$is suppressed after the reaction (Figure 1). Then, an aqueous solution of PEI (polỵ ethy lenimine. $\mathrm{MW}=400.2 \mathrm{~g} / \mathrm{L}, \mathrm{pH}=9$ ) was added to the suspension of platinum nanoparticles. Then, the reaction was carried out under vigorous stirring. In this way, the adsorption of PEI polymers provided the Pt nanocry'stals with positive surface charge. leading to the effective adhesion of the Pt nanoparticles onto the surface of titanium oxide. ${ }^{1+4}$ as illustrated in Figure 2.

Synthesis of the Restacked Assembly of Titanate with Pt Nanoparticles. To prepare the restacked assembly of titanate with Pt nanoparticles. we added small amount of Pt suspension into the colloidal suspensions of exfoliated titanite nanosheets, leading to the formation of opalescent suspension with milky brown color. The reaction proceeded for $18 \mathrm{~h}$. After the reaction. viscous brown powder of Pt-loaded titanium oxide was collected from the reactant suspension via centrifugation

Pt-loading for Protonated Titanate. To probe the effect of mesopore formation on the photocatalytic activity of the layered titanate, we have prepared the Pt-loaded protonated titanate as a reference. Due to the negligible surface charge of the protonated titanate. it is very difficult to load positive-char-

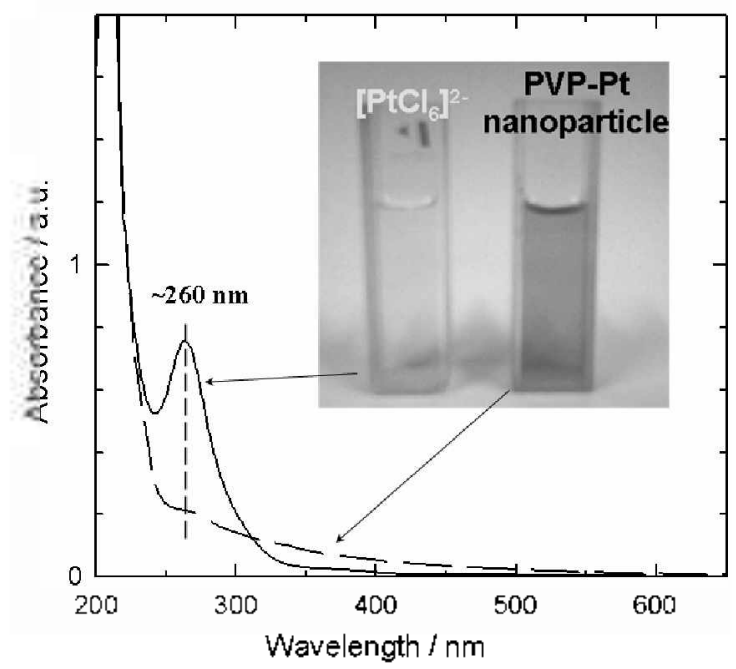

Figure 1. UV-vis spectra of the reactant $\left[\mathrm{PtCl}_{\mathrm{r}}\right]^{2 \cdot}$ (solid line) and PVP-coated Pt nanoparticle (dashed lines). Inset: photographs for the color change upon the formation of Pt nanoparticles.

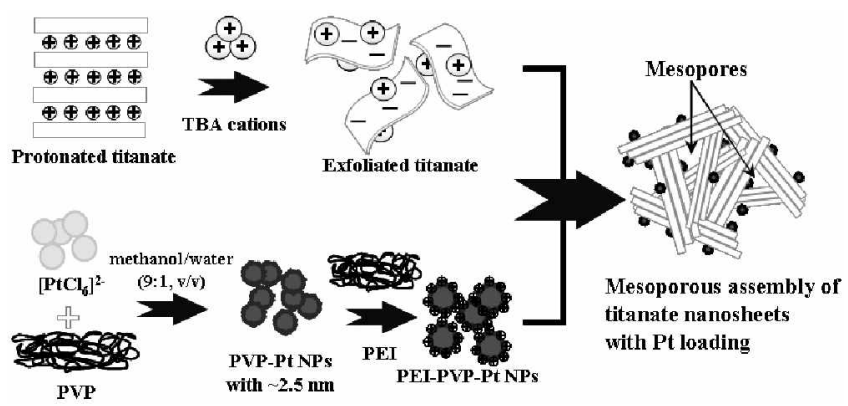

Figure 2. Schematic model for the restacking of exfoliated titanate nanosheets with the adsorption of Pt nanoparticles. ged Pt nanoparticles onto the surface of the protonated titanate. For this reason. we have carried out the Pt-loading for the protonated titanate through a photoreductive deposition of Pt-containing precursor: ${ }^{15}$ Pt loading for the protonated titanate was carried out in an aqueous mixture of the protonated titanate $(0.5 \mathrm{~g} / \mathrm{L}) .1 \mathrm{M}$ methanol. and $0.1 \mathrm{mM}$ chloroplatinic acid $\left(\mathrm{H}_{2} \mathrm{PtCl}_{\hat{\sigma}}\right)$ under UV illumination (400 W-Oriel UV-Xenon lamp). After illumination for l h. Pt-loaded protonated titanate was filtered. washed with distilled water, and dried under air.

Sample Characterization. The crystal structures of the present samples were examined by powder XRD analysis with a Rigaku D/Max-2000/PC general X-ray diffractometer using $\mathrm{Cu} \mathrm{Ka}$ radiation. The crystal morphology and chemical composition of the samples were probed by HR-TEM (JEOL JEM-2100F) and FE-SEM (JEOL VSM-6700F) equipped with energy dispersive spectrometry (EDS). The surface area and porosity of the titanates were determined by measuring volumetrically $\mathrm{N}_{2}$ adsorption-desorption isotherms at liquid nitrogen temperature. The calcined samples were degassed at $150^{\circ} \mathrm{C}$ for $2 \mathrm{~h}$ under vacuum before the adsorption measurement. Photocatalytic measurements were carried out in a Pyrex photoreactor $(100 \mathrm{~nL})$ with a quartz window. A $400 \mathrm{~W}$-Oriel UV-Xenon lamp filtered below $\lambda<290 \mathrm{~nm}$ was used as the light source. and MB was used as a test substrate. The substrate $(50 \mu \mathrm{M})$ was added to an aqueous suspension of the photocatalyst $(0.5 \mathrm{~g} / \mathrm{L})$ in the glass reactor $(100 \mathrm{~mL})$ with a quartz window and then equilibrated for 30 min with stirring in the dark before illumination. All the tests of photocatalytic reactivity were carried out in aerobic condition. Sante aliquots were withdrawn intermittently with a $5 \mathrm{~mL}$ syringe during the illumination and filtered through a $0.45 \mu \mathrm{m}$ PTFE filter (Milipore) to remove catalyst particles. The concentration change of $\mathrm{MB}$ was monitored spectrophotometrically by measuring the absorbance at $\lambda=665 \mathrm{~nm}$ with an UV-vis spectrophotometer. ${ }^{16}$ The variation of MB concentration was measured for the reaction periods of $20.40 .60 .120,180.240$, 300.360 . and $420 \mathrm{~min}$.

\section{Results and Discussion}

Powder XRD Analysis. Figure 3 represents the powder XRD patterns for the cesium titanate. the protonated titanate. the exfoliated colloidal titanate. and the restacked titanate. Both the XRD patterns of the cesium titanate and its protonated derivative could be well-indexed with orthorhombic lepidocrocite structure. Upon protonation. the basal spacing of the pristine cesium titanate slightly expands. indicating the intercalation of hydronium ion $\left(\mathrm{H}_{3} \mathrm{O}^{+}\right)$into the interlayer space of layered titanate. As shown in Figure 3. the exfoliated nanosheets of layered titanate did not show any sharp Bragg reflections, indicating the separation of the $3 \mathrm{D}$ lattice of layered titanate into individual monolayers. Similarly, no well-developed Bragg reflections could be observed for the restacked assembly of layered titanate, reflecting the disordered stacking of layered titante. Either, no evidence for the intercalation of Pt nanoparticles into the interlayer space of the layered titanate could be obtained. A closer inspection on the XRD 
data of the restacked titanate revealed that a small but distinct peak corresponding to $(200)$ reflection of the layered titanate appears at $2 \theta=\sim 48^{\circ}$. This finding can be regarded as evidence for the maintenance of in-plane structure of the layered titanate even after the restacking process. It is worthwhile to note here that the absence of the platinum-related peaks in the XRD pattern of the restacked titanate clearly demonstrates the

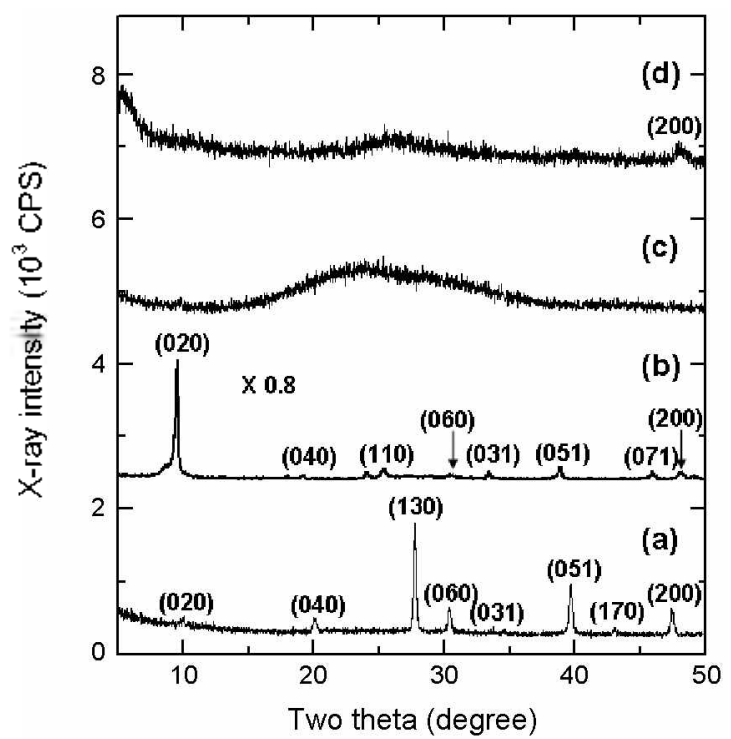

Figure 3. Powder XRD pattens of (a) the pristine cesimn titantate. (b) the protonated titanate, (c) the exfoliated colloidal suspension, and (d) the restacked assembly of layered titanate with Pt loading.
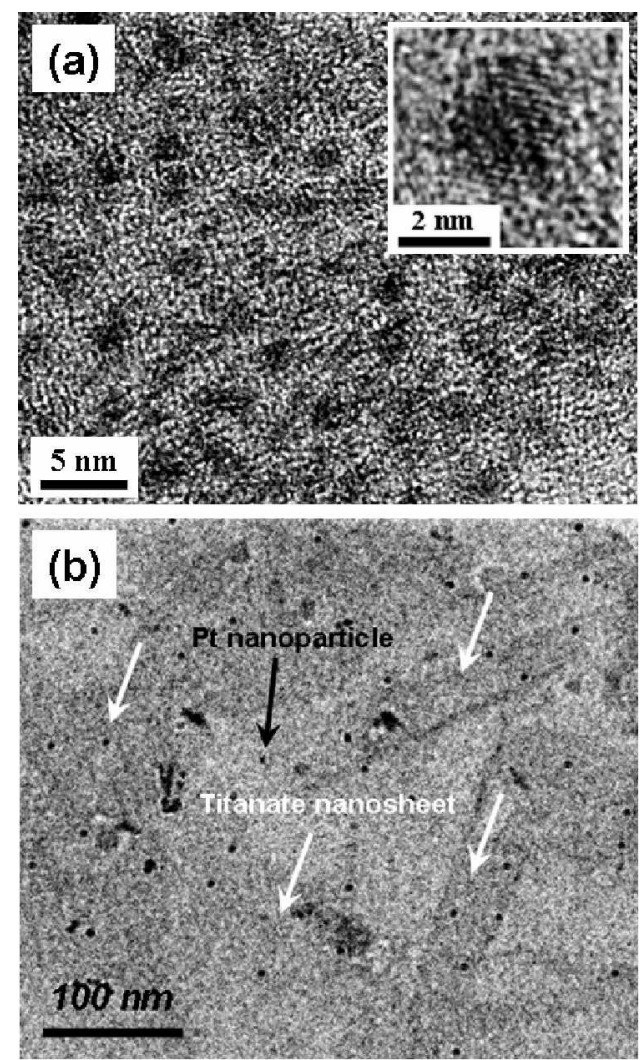

Figure 4. HR-TEM inages of (a) the PVP-Pt nanoparticles and (b) the restacked assembly of lavered titanate with $\mathrm{Pt}$ loading. homogeneous dispersion of Pt nanoparticles amongst the titanate nanosheets. The present XRD result strongly suggests that the exfoliated titanate nanosheets are restacked in disordered way and the platinum nanoparticles are well-embedded in the porous assembly of the layered titanate. as shown in Figure 2.

HR-TEM, FE-SEM, and Elemental Mapping Analysis. The crystal norphology of the Pt nanoparticles and the restacked titanate with $\mathrm{Pt}$ loading was studied with HR-TEM technique. As shown in Figure ta. the platimum particles have very uniform particle size of $\sim 2.5 \mathrm{~nm}$. After the reaction with layered titanate nanosheets, the platinum nanoparticles were well-loaded on the surface of layered titanate crystallites without significant agglomeration (Figure $4 b$ ). We have also examined the crystal shape of the restacked titanate using FE-SEM techuique. Figure 5 illustrates the FE-SEM intage of the restacked assembly of the layered titanate. The FE-SEM result presented here strongly suggests that porous structure is formed by the house-of-cards type stacking of layered titanate crystallites. On the other hand. a chenucal composition and a

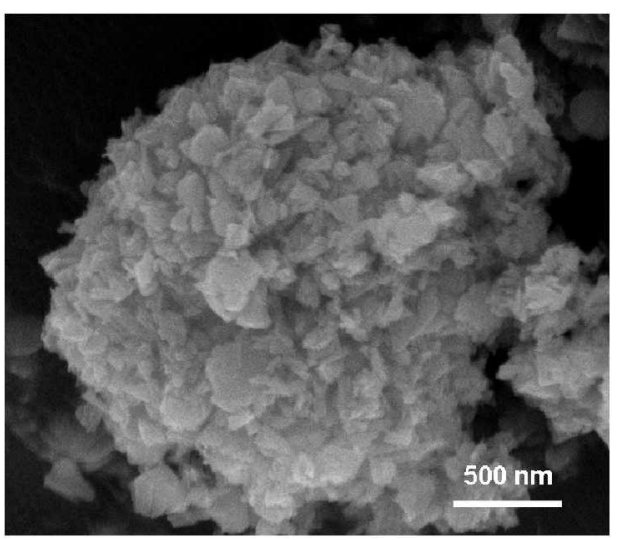

Figure 5. FE-SEM inage of the restacked assembly of lavered titanate with $\mathrm{Pt}$ loading.

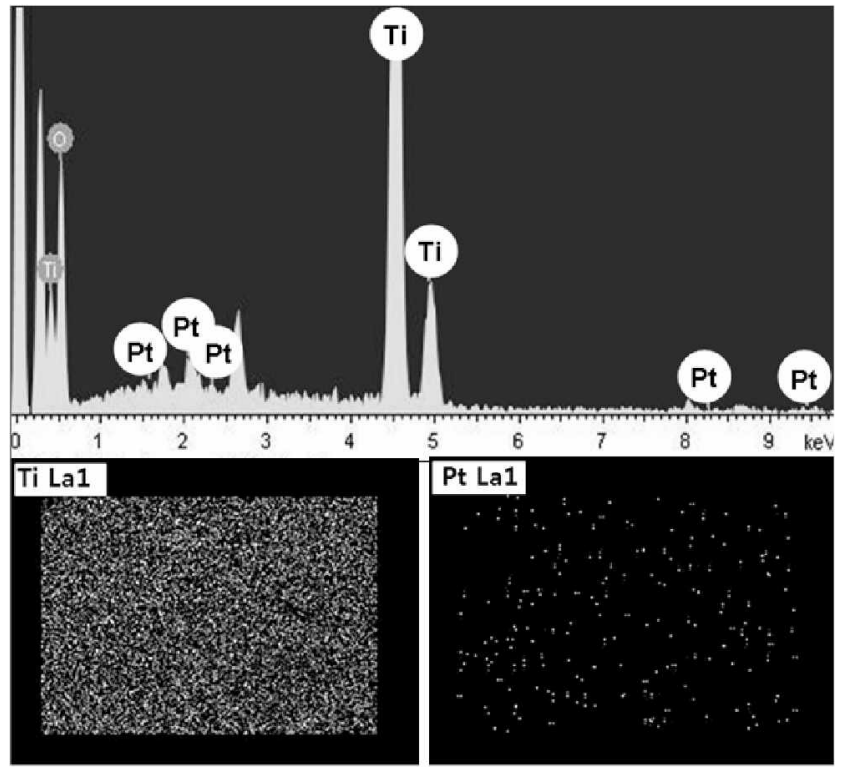

Figure 6. (Top) EDS data and (bottom) elemental maps of the restacked assembly of layered titanate with Pt loading. 
spatial distribution of Pt cocatalysts in the restacked titanate with Pt-loading were determined with EDS and elemental mapping analysis. respectively. As plotted in the EDS spectrum of Figure 6, the restacked assembly of the layered titanate contains small amount of Pt nanoparticles. From the quantitative analysis, the amounts of platinum loaded in restacked titanate was estimated to be $2.12 \pm 0.02 \mathrm{wt} \%$. which is typical amount of Pt cocatalyst loaded on the metal oxide photocatalysts. ${ }^{15}$ In addition, the elemental mapping analy sis indicated that Pt as well as Ti elements are homogeneously distributed in the restacked titanate (the bottom panel of Figure 6). This provides strong evidence for the non-aggregated dispersion of Pt cocatalysts in the porous assembly of the layered titanate.

$\mathbf{N}_{2}$ Adsorption-Desorption Isothem Measurements. We have studied the pore structure of the present restacked titante by measuring $\mathrm{N}_{2}$ adsorption-desorption isotherm. Figure 7 plots the $\mathrm{N}_{2}$ adsorption-desorption isothenns of the Pt-unloaded protonated titanate and the Pt-loaded restacked titanate. In case of the isotherm of the restacked compound a hysteresis is distinctly observed in the pressure region of $\mathrm{p} / \mathrm{p}$ underscoring the presence of mesopores or macropores formed by the house-of-cards stacking of the layered crystallites. Similar type of isothern was reported for the titanate-based mesoporous materials. "The observed hysteresis behavior could be classified as the Brunauer-Deming-Deming-Teller (BDDT) type I and IV shape. along with H3-type hysteresis loop in the IUPAC classification. ${ }^{18}$ This finding can be interpreted as proof for the presence of the open slit-shaped capillaries with very wide bodies and narrow short necks. The surface area of the restacked titanate $\left(-67 \mathrm{~m}^{3} / \mathrm{g}\right)$ is about 5 times larger than that of the protonated titanate $\left(\sim 13 \mathrm{~m}^{\hat{2}} / \mathrm{g}\right)$. underscoring the expansion of surface area upon the restacking process. Based on Barrett-Joyner-Halenda (BJH) equation. we have determined the mean pore diameter of the restacked titanate as $\sim 14 \mathrm{~nm}$.

Test of Photocatalytic Activity. The photocatalytic activity of the restacked titanate with well-dispersed Pt cocataly st was tested by monitoring the photodegradation of MB molecules. in comparison with that of the protonated titanate with $\mathrm{Pt}$

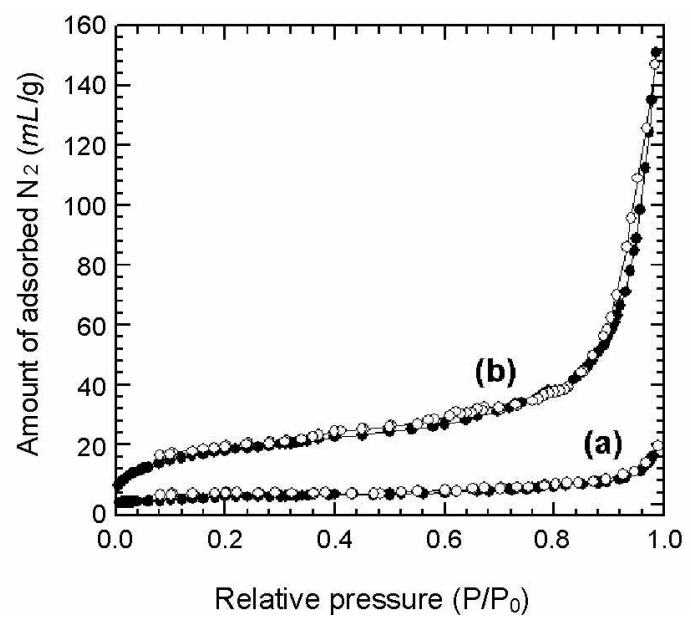

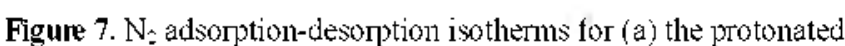
titanate and (b) the restacked assembly of layered titanate with $\mathrm{Pt}$ loading.

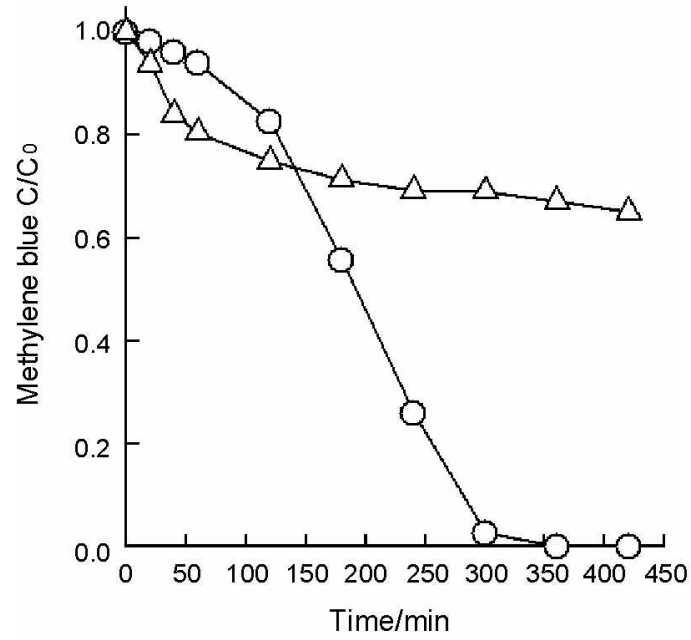

Figure 8. Time profiles of photocataly tic degradation of MB in UV-vis illuminated $(\lambda>290 \mathrm{~nm})$ suspensions of the restacked assembly of lavered titanate with Pt loading (circles) and the protonated titanate with Pt loading (triangles).

loading. It was well-known that the loading of Pt cocatalysts enlances the life-time of hole through the spatial separation of excited electron leading to the inprovement of the photocatalytic activity. ${ }^{15}$ The EDS analysis clearly demonstrated that the reference of Pt-loaded protonated titanate contains $2.30 \pm$ $0.05 \mathrm{wt} \%$ of Pt cocatalyst. which is almost identical to the amount of Pt loaded in the restacked titanate (2.12\%). Since an intercalation of Pt nanocrystals into neither the protonated titanate nor restacked one was evidenced by XRD analy sis. the Pt cocataly sts are believed to exist on the surface sites of both the titanates. Hence. we can rule out possible variations in the photocatalytic activities of the protonated and restacked titanates caused by difference in the content or loading site of $\mathrm{Pt}$ cocataly sts. Figure 8 plots the time-dependent variation of relative $\mathrm{MB}$ concentration during the UV-vis illumination in the presence of the photocatalysts. In contrast to an incomplete decomposition of $\mathrm{MB}$ by the protonated titanate. the restacked titanate photodegrades all the MB molecules within $5 \mathrm{~h}$, underscoring its higher photocatalytic activity. It is certain that the photocatalytic activity of the layered titanate becomes much improved upon the formation of mesoporous structure. Of special interest is that the restacked titanate with Pt-loading causes a slower photodegradation of MB than the protonated titanate with Pt-loading within $\sim 150 \mathrm{~min}$. Such a weak photocatalytic activity of the restacked titanate at the initial stage of photoreaction can be rationalized from the fact that the hybridized platinum nanoparticles contain significant amount of polymer on their surface and hence the photocatalyst first photodegrades coated PVP and PEI species rather than the $\mathrm{MB}$ substrate. After the removal of surface-coated polymer, an electronic connection between Pt cocatalysts and layered titanate becomes much improved, leading to the remarkable enhancement of photocatalytic activity after $150 \mathrm{~min}$.

\section{Conclusion}

We have clearly demonstrated that mesoporous titanate 
with well-dispersed Pt cocatalyst can be prepared by the restacking of exfoliated titanate nanosheets and the simultaneous loading of Pt nanoparticles. The crystal strncture. morphology, and elemental composition of the restacked assembly of the layered titanate were characterized by powder XRD, HR-TEM. FE-SEM. and EDS/elemental mapping analy sis. Overall characterizations clearly demonstrated that the exfoliated titanate nanosheets are restacked in disordered way and the Pt nanoparticles are well-dispersed in the porous assembly of the titanate. The formation of mesoporous stnicture due to the house-of-cards type stacking of the titanite crystallites was evidenced by $\mathrm{N}_{z}$ adsorption-desorption isotherm measurements. The mesoporous assembly of Pt-loaded titanate showed a higher photocatalytic activity than the protonated titanate with Pt loading. Taking into account similar contents and similar loading sites of Pt cocatalysts in both the protonated and restacked titanates. the present finding underscores that the expansion of surface area upon the restacking of the layered titanate play's a main role in increasing the photoefficiency of layered titanate.

Acknowledgments. This work was performed for the Hy'drogen Energy R\&D Center. one of the 21st Century Frontier R\&D Program. fiunded by the Ministry of Science and Technology of Korea and supported partly by the SRC/ERC program of MOST/KOSEF (grant : R1 l-2005-008-03002-0).

\section{References}

1. Honda, K. Fujishima. A. Nature 1972.238. 37

2. Hoftmann, M. R: Martin, S. T.; Choi, W.: Bahnemann, D. W. Chem. Rev 1995, 95, 69 .

3. Oh, W.-C.; Chen, M.-L. Bull. Kon Chen. Soc. 2008, $29,159$.

4. Kim, S. W.: Khan, R.: Kim, T.-T.; Kim, W.-T. Bull Kor Chem. Soc. 2008, 29, 1217

5. Anpo, M. Bull. Chem. Soc. Jph 2004, 77, 1427.

6. Kim S.: Hwang. S.-I.: Choi, W.J. Phis Chem. B 2005, 109, 24260.

7. Kim, T. W.; Hwang, S.-J.; Juung, S. H.; Chang, J.S.; Park, H; Chol, W. Choy, I-H. Adv Mater. $2008,20,539$

8. Kim, T. W . Hur, S. G.; Hwang, S.-T. Park, H.: Choi, W.; Choy, T.-H. Adw Funct Mater $\mathbf{2 0 0 7}, 17,307$.

9. Kim, T. W. Hur, S. G.: Hwang, S.-T.: Choy, J-H. Chem Commm. 2006,220 .

10. Kim, T. W.; Hwang, S.-J.; Park, Y.; Choi, W.; Choy, J.H. J. Phys Chem C 2007, 111, 1658.

11. Park, H. M. Kim, T. W. Hwang, S.-J-; Choy, J.-H. Bull Kor: Chem. Soc. 2006, 27,1323 .

12. Kim, T. W.: Ha, H.-W.: Paek, M.-J.; Hyun, S.-H.; Choy, J.-H.; Hwang, S.-J. J. Phys. Chem. C 2008, 112, 14853.

13. Teranishi, T: Hosoe, M.: Tanaka, T.: Miyake, M. J. Phys Chem. $\mathrm{B} 1999,103,3818$.

14. Zhou, Y: Ma, R.: Ebina, Y: Takada, K.: Sasaki, T. Chem. later $2006,18,1235$.

15. Bae, E. Choi, W. Park, J-; Shin, H. S.; Kim, S. B.; Lee J. S. J. Phis. Chem. $2004,108,14093$.

16. Hur, S. G.; Kim, T. W.; Hivang, S.-J.; Choy, J.-H. J. Photochem. Photobiol . A Chem 2006, 183,176 .

17. Sasaki, T.; Nakano, S.; Yamauchi, S.; Watanabe, M. Chem. Hater. $1997,9,602$.

18. Gregg, S. I. Sing, K. S. W. In Adsorption. Surface Area and Porosity, Acadenic Press: London, 1976. 